\section{H I G H L I G H TS}

CYTOSKELETON

\section{Space invaders}

The hallmarks of Rho-family-GTPase activation - stress fibres, lamellipodia and filopodia - require the Wiskott-Aldrichsyndrome protein (WASP) family and, of course, the actin-nucleating Arp2/3 complex. Cdc42 interacts directly with the WASP-family protein N-WASP to stimulate actin polymerization, but no such direct binding has been detected between Rac and the WASPrelated protein WAVE. Indeed, unlike WASP, WAVE lacks a Cdc42/Rac-interactive binding (CRIB) motif, so how it functions downstream of Rac to induce membrane ruffles has been unclear. Now, however, research from Marc Kirschner's laboratory provides an explanation of how Rac and the adaptor protein Nck activate actin nucleation. In contrast to N-WASP, which is autoinhibited by an intramolecular interaction between the carboxyl and amino termini, recombinant WAVE1 can constitutively induce actin nucleation by

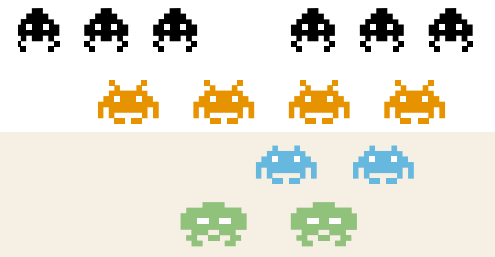

Arp2/3. To prevent actin nucleation occurring without external signals, WAVE activity in vivo must either be inhibited by other proteins or regulated post-

translationally. To investigate the former possibility, Kirschner and colleagues looked for WAVE-interacting proteins, and used size-exclusion chromatography to analyse a protein complex from bovine brain extracts in which WAVE1 elutes. They identified four proteins in addition to WAVE1: a p53inducible messenger RNA with a relative molecular mass of 140,000 (PIR121); a NCKassociated protein with a relative molecular mass of 125,000 (Nap125); a protein similar to hNap1-binding protein; and HSPC300.

The complex does not activate the Arp $2 / 3$ complex in vitro. Adding GTP $\gamma S$-bound Rac1 (a non-hydrolysable form of GTP), however, does stimulate the activity of Arp2/3, suggesting that Rac1 can relieve WAVE1 inhibition in the complex. Both Nap125 and PIR121 can associate with NCK, so the authors tested whether NCK could induce

\title{
ESCRTs needed
}

ESCRT ('endosomal sorting complex required for transport') protein complexes are required to sort ubiquitylated endosomal membrane proteins into the multivesicular body (MVB) pathway. This pathway, which leads to the vacuolar/lysosomal lumen, has a crucial role in sorting and downregulating/degrading activated cell-surface receptors. In addition, the ESCRT complexes have recently been shown to be required for the budding and release of retroviruses such as HIV. A detailed understanding of the molecular machinery for MVB sorting has been lacking, but, in two papers in Developmental Cell, Scott Emr and colleagues now report the characterization of two of the ESCRT complexes - ESCRT-II and ESCRT-III.

Vps24 and Snf7 — class E vacuolar protein sorting (Vps) proteins - had previously been shown to be part of an endosome-associated protein complex ('class E' refers to the 'class E compartments', large endosomal structures that accumulate in class $\mathrm{E} v p s$ mutants). In the first of their two studies, the authors searched for additional components of this complex, and identified Vps2 and Vps20. They found that Vps24, Snf7, Vps2 and Vps20 exist in the cytoplasm in a soluble, monomeric form, but that these proteins can be recruited to the endosomal membrane, where they form an oligomeric protein complex called ESCRT-III.

By further analysing ESCRT-III, Emr and coworkers showed that it contains two functionally distinct subcomplexes - Vps2-Vps24 and Vps20-Snf7. Dissociation of the ESCRT-III complex depends on the AAA-type ATPase Vps4, and they showed that Vps4 binds to ESCRT-III through interactions with Vps2-Vps24. They found that the Vps20-Snf7 subcomplex, on the other hand, is responsible for the membrane association of ESCRT-III, and that it might also have a direct role in the sorting and/or concentration of MVB cargo.

In the second study, Emr and colleagues showed that ESCRT-II is composed of three class E Vps proteins (Vps22, Vps25 and Vps36), and that it is required for the membrane recruitment and assembly of ESCRT-III. They found that ESCRT-II in the cytoplasm associates transiently with endosomal compartments, where it interacts with ESCRT-III through Vps20. They also showed that ESCRT-II functions downstream of ESCRT-I, and that ESCRT-II release from the membrane depends on Vps4.

These studies have provided valuable insights into the molecular machinery used for MVB sorting, and have enabled the authors to propose a model for the sorting of ubiquitylated cargo. First, ESCRT-I — which binds ubiquitylated
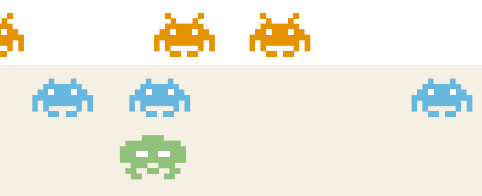

the WAVE1 complex to activate Arp2/3, which it could. Furthermore, GTP $\gamma \mathrm{S}-\mathrm{Rac1}$ and NCK activated the WAVE1 complex additively, but, because neither could induce additional activation of recombinant WAVE1, the authors proposed that they function to relieve inhibition rather than to stimulate activity.

This being the case, Racl and NCK might be able to induce dissociation of the complex to release active WAVE1. The complex indeed disassembled into two subcomplexes. Because the fraction that contained WAVE1 (WAVE1-HSPC300) stimulated Arp2/3dependent actin nucleation, the scenario seems to be that Racl or NCK can induce the formation of lamellipodia/membrane ruffles by disassembling the trans-inhibited WAVE complex, leaving WAVE free to go about its duties.

\section{(D) References and links}

Katrin Bussell ORIGINAL RESEARCH PAPER Eden, S. et al. Mechanism of regulation of WAVE1-induced actin nucleation by Rac1 and Nck. Nature 418, 790-793 (2002)

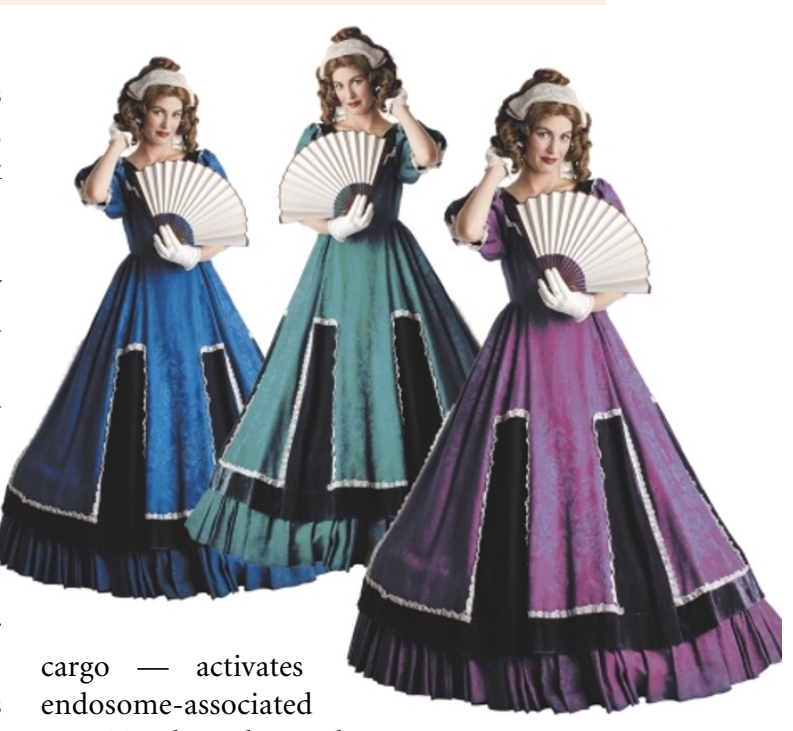

endosome-associated

ESCRT-II through a mecha-

nism that is not yet clear. Activated ESCRT-II then recruits and assembles ESCRT-III by binding to Vps20, and the formation of the ESCRT structure results in the concentration and sorting of MVB-pathway cargo. Finally, after sorting is complete, ESCRT-III recruits Vps4, which results in the dissociation and disassembly of the ESCRT structure.

\section{6) References and links}

Rachel Smallridge

ORIGINAL RESEARCH PAPERS Babst, M. et al. ESCRT-III: an endosome-associated heterooligomeric protein complex required for MVB sorting. Dev. Cell 3, 271-282 (2002) | Babst, M. et al. Endosome-associated complex, ESCRT-II, recruits transport machinery for protein sorting at the multivesicular body. Dev. Cell 3, 283-289 (2002) 